\title{
ELECTRIC POLLUTION STUDIES IN MESH TYPE MTDC SYSTEM USING NEURAL NETWORK
}

\author{
K.G.Narendra H.S.Chandrasekharaiah \\ Department of High Voltage Engineering \\ INDIAN INSTITUTE OF SCIENCE \\ BANGALORE,INDIA
}

\begin{abstract}
In this paper we are proposing a Neural Network identifier to estimate the electric pollution (harmonics) contents present in the voltage and current signals of a mesh type Multi Terminal Direct Current (MTDC) system under dynamic conditions. A digital computer program has been developed to implement the Neural Network and a modified form of Fourier series representation which improves the accuracy of the results is discussed.
\end{abstract}

Key Words: MTDC system, Neural Network, Harmonics

\section{INTRODUCTION}

The purpose of this paper is to conduct electric pollution studies in a mesh type MTDC system using Neural Network. This work was formulated since many two terminal HVDC stations are being converted into MTDC systems and a study of the dynamic interaction of harmonics in such systems is very helpful for designing of filters, whose cost affect significantly the economy of the overall system. Limited literature is available on the electric pollution analysis in MTDC systems [1]. In India also, MTDC system has potential future in meeting the growing power transmission requirements.

A digital computer program has been developed for dynamic analysis of a four terminal MTDC system with long $D C$ lines connected in mesh with two inverters and two rectifiers transmitting $500 \mathrm{MW}$ power at $500 \mathrm{kV}$ potential[4]. The results obtained are fed to the Neural Network for estimation of the harmonic content (electric pollution) present in the signal. The concept used here is that a given signal can be represented by a finite number of sinusoids in the form of trignometric polynomial over the fundamental range. This yields a set of simultaneous linear equations. A matrix method has been developed to convert the overdetermined equations to a well defined positive definite matrix. A general steep gradient approach is used to express the matrix differential equation whose steady state solution yields the required harmonic coefficient of the polluted signal. The advantage of the method is that the concept developed can be applied for real time studies under various operating conditions of the system.

\section{NEURAL NETWORK (NN) DEVELOPMENT}

The Fourier series representation to approximate a given function, which satisfies the Dirichlet's conditions is a series with trignometric polynomials which are orthogonal over the fundamental range. This can be expressed as:

$$
b(t)=X_{0}+\sum_{r=1}^{q}\left(X_{r} \cdot \sin (\omega r t)+Y_{r} \cdot \cos (\omega r t)\right)
$$

Over one fundamental period, equation (1) can be written for different time intervals can be written in the matrix notation as

$$
\left.\left.[A]_{\mathbf{n x}(2 q+1)} \cdot \mathbf{x}\right]_{(2 q+1) \times 1}=b\right]_{\mathbf{n x 1}}
$$

where, $[\mathbf{A}]$ is the matrix of sinusoids ( sine and cosine terms), $\mathbf{x}]$ is the coefficient vector which is the unknown, and b] is the actual signal.

By pre-multiplying the equation(2) by $\left[\mathbf{A}^{\mathbf{T}}\right]$, it is reduced to,

$$
[\mathbf{G}] \cdot \mathbf{x}]=\mathbf{d}]
$$


where $[\mathbf{G}]=\left[\mathbf{A}^{\mathbf{T}}\right] \cdot[\mathbf{A}]$, and $\left.\left.\mathbf{d}\right]=\left[\mathbf{A}^{\mathbf{T}}\right] \cdot \mathbf{b}\right]$. Equation (3) is the normal form of representing the overdetermined set of linear equations into a pseudo invertable matrix. It is shown in reference [2] that matrix [G] can be reduced to a diagonal matrix which is symmetric and positive definite. For analog representations which can give parallel solution in real time neural optimization principle is used which allows us to represent the matrix differential equation as:

$$
\mathrm{d}[\mathbf{x}] / \mathrm{dt}=-\mathbf{h} .([\mathbf{G}] \cdot \mathbf{x}]-\mathrm{d}])
$$

with $\mathbf{x}(\mathbf{0})=\mathbf{x}^{\mathbf{0}}$ and $\mathbf{h}$ is a scalar.

As described in [3] with [G] positive definite and symmetric, equation (4) is equivalent to the unconstrained quadratic programming problem which gives global minimum solution of the energy function.

\section{COMPUTATIONAL ANALY- SIS}

The required harmonic components can be calculated selectively because [G] is diagonal.

The differential equation (4) is reduced to the standard state space form as:

$$
\dot{y}=[H] \cdot y]+[B] . u]
$$

where, $[H]=[G], y]=x], \quad[B]=\operatorname{diag}(1)$ and $u]=$ $\left.\left[A^{T}\right] . b\right]$. The steady state solution of (5) gives the required harmonic component. After evaluating the coefficients $X_{0}, X_{1}$ and $Y_{1}$, as discussed in [2], the Fourier series is described as:

$b(t)-X_{0}-X_{1} \cdot \sin (\omega t)-Y_{1} \cdot \cos (\omega t)=$

$$
\sum_{r=2}^{q}\left(X_{r} \cdot \sin (\omega r t)+Y_{r} \cdot \cos (\omega r t)\right)
$$

which can be written as:

$$
b(t)_{-1}=\sum_{r=2}^{q}\left(X_{r} \cdot \sin (\omega r t)+Y_{r} \cdot \cos (\omega r t)\right)
$$

and $b(t)_{-1}=b(t)-X_{0}-X_{1} \cdot \sin (\omega t)-Y_{1} \cdot \cos (\omega t)$ is the modified representation of the function by filtering the fundamental and dc harmonic contents from the signal. Similarly, while calculating the $3^{\text {rd }}$ harmonic component,

$$
b(t)_{-2}=\sum_{r=3}^{q}\left(X_{r} \cdot \sin (\omega r t)+Y_{r} \cdot \cos (\omega r t)\right)
$$

is used. In general, for finding $p^{\text {th }}$ harmonic component the equation is written as:

$$
b(t)_{-(p-1)}=\sum_{r=p}^{q}\left(X_{r} \cdot \sin (\omega r t)+Y_{r} \cdot \cos (\omega r t)\right)
$$

This representation has improved the numerical results to a great extent because the neural method matches only the un-calculated coefficients with that of the signal which does not contain the calculated harmonics [2].

\section{BRIEF DETAILS OF THE MTDC SYSTEM}

Four terminal dc system considered for the study is shown in fig.(A). It consists of mesh type parallel monopolar dc system connecting four ac systems by means of long dc lines of two 400,600 and $800 \mathrm{kM}$ length. The converter system comprises of two rectifier and two inverter station, each is a 12-pulse bridge operating as two 6-pulse bridges in series. The dc power transmitted by each station is $500 \mathrm{MW}$ at $1 \mathrm{kA}$ current and $500 \mathrm{kV}$ potential. Each ac system consists of tuned filters $\left(5^{\text {th }}, 7^{\text {th }}, 11^{\text {th }}, 13^{\text {th }}\right.$ and HP) and a static load (15\%). Conventional PI current controller is used at each rectifier station and Minimum Extinction Angle (MEA) controller is used at each inverter station. For overall current order coordination, a Constant Current Margin (CCM) controller is employed. The fundamental frequency of operation is at $50 \mathrm{~Hz}$. The sampling rate is considered at $10 \mathrm{kHz}$ which is sufficient for the dynamic analysis. The simulation of the MTDC system is done by the method as explained in reference [4].

\section{NUMERICAL RESULTS AND DISCUSSION}

Digital simulation of the MTDC system described is carried out and the variables are then fed to the Neural Network to detect the required harmonic component from the electrically polluted wave form. In the fig. 1, the electric pollution studies of the source current under various system contingencies is shown. Harmonics present in the source current of the 'a' phase of a rectifier1 under steady state condition is depicted in fig 1a. It can be observed that, the convergence of all the harmonics $(1-20)$ is achieved in only two steps in almost super real time ( 0.1 microsecond). Phase variation under this condition is shown in fig. $1 b$. Following case studies were carried out:

1) Change in the system frequency from $50 \mathrm{~Hz}$ to $48.5 \mathrm{~Hz}$ after the two cycles from the steady state. 2) The transformer reactance of the upper bridge of the rectifier 1 and inverter 2 were increased by about $5 \%$.

3) A $5 \%$ negative sequence voltage was added to 
all the ac-sources.

The variation of harmonic magnitude and phase is illustrated in figs $1 \mathrm{c}, 1 \mathrm{~d}, 1 \mathrm{e}, 1 \mathrm{f}, 1 \mathrm{~g}, 1 \mathrm{~h}$ respectively for the three cases stated. The mark '*' in all the figures represents the $1 \%$ magnitude barrier of the fundamental. Only the $11^{\text {th }}$ harmonic current is above this barrier. But significant phase pollution can be observed in all the cases. In fig. 2 , the dcside harmonic spectrum is depicted in which fig. 2 a correspond to steady state condition and fig. $2.1 \mathrm{~b}$ correspond to case 1 . Higher order harmonics are significantly damped out in the case 1 which.can be due to the shift in the crossover point which has decreased firing angle and hence smoother response is observed (see fig. 2.1a from 0.02 to 0.04 second).

Further, a solid ground fault is created on the ac side of 'a' phase bus at the inverter2 (fig. 3a) from 0.015 to 0.02 second. The wave form is polluted after the system starts recovering and significant $10^{\text {th }}$ harmonic is observed. In fig. $3 \mathrm{~b}$ the magnitude spectrum for 20 harmonics over five cycles is illustrated which shows the presence of the uncharacteristic $10^{\text {th }}$ harmonic, whose magnitude decreases with time.

For the same case on the dc side, electric pollution chrateristics of the rectifier $1 \mathrm{dc}$ voltage over four cycles is shown in fig. 4. In all these figures, ' $6 p$ ' represents the charateristic harmonic, plotted from 0 to 10 on the $x$-axis, uncharateristic harmonics ' $6 p+1$ ' is plotted from 11 to 20 and ' $6 p-1$ ' from 20 to 30 , with $p$ an integer varying from 1 to 10 . As shown the uncharateristic $10^{\text {th }}$ harmonic on the ac side, as discussed has now appeared on the dc-side as $11^{\text {th }}$ harmonic with significant magnitude.

\section{CONCLUSIONS}

A Neural Network for fast-evaluation of the harmonic components present in the eletrically

Fig.(A) MTDC-AC

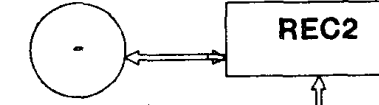
AC system SYSTEM CONSIDERED FOR THE STUDY polluted signal of MTDC system is developed. From the studies it is observed that the phase pollution is more significant even under small disturbances which is normally ignored. Uncharateristic harmonics of significant magnitude play a dominant role over few cycles under the fault condition. It can be said that the knowledge of both the magnitude and phase of harmonic is a better criteria than taking only the magnitude for designing of filters and control parameters.

\section{ACKNOWLEDGEMENTS}

The authors would like to thank Prof. G.R. Nagabhushana, Chairman, department of High Voltage Engineering for his encouragement in this work.

\section{REFERENCES}

1 Melvod, D.J., T.Endo, and H.P. Lips, "Effects of operating configurations of Multiterminal HVDC Systems on DC filter performance",IEEE Trans., PWRD, Jul-91, p 924930.

2 K. G. Narendra, H. S. Chandrasekahraiah, "A novel neural architecture for harmonic identification in an integrated ac-dc power system", (paper submitted for IEEE, P.E.S., SM.)

3 A. Cichocki and R. Unbehauen,"Neural Networks for Solving Systems of Linear Equations and Related Problems", IEEE Trans. Cir-Sys-1: Fundamental Theory and Applications, Vol. 39, No. 2, Feb-92, p 124-138.

4 Manohar P. and H.S. Chandrasekharaih,"Artificial commutation for inversion into weak ac system in multiterminal HVDC system", J. EPSR, (U.K.), Vol. 19,

1990 p95-104.'
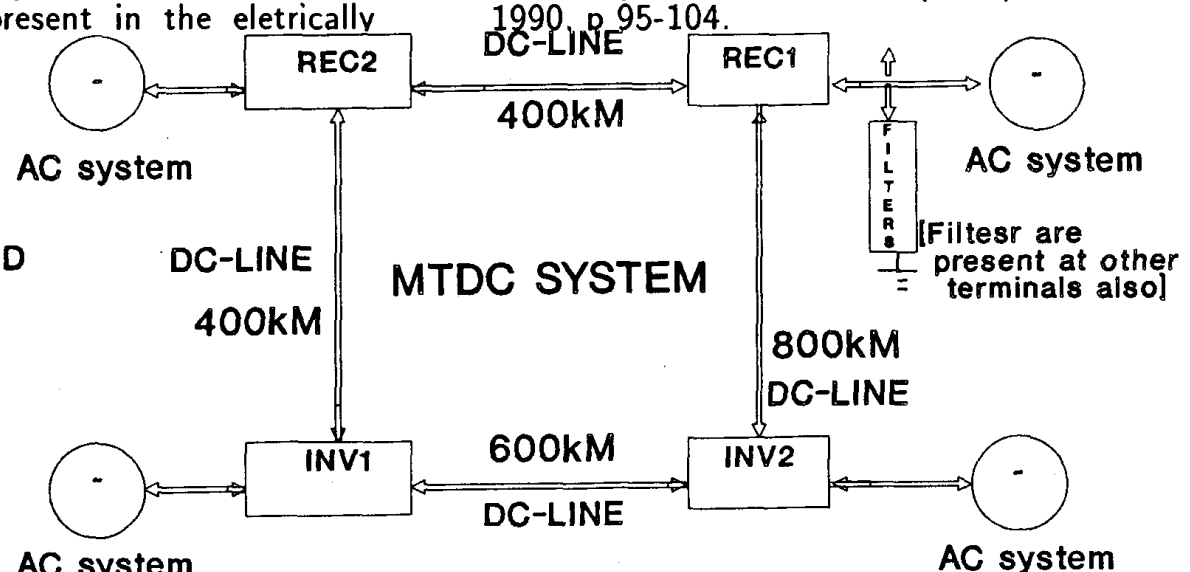

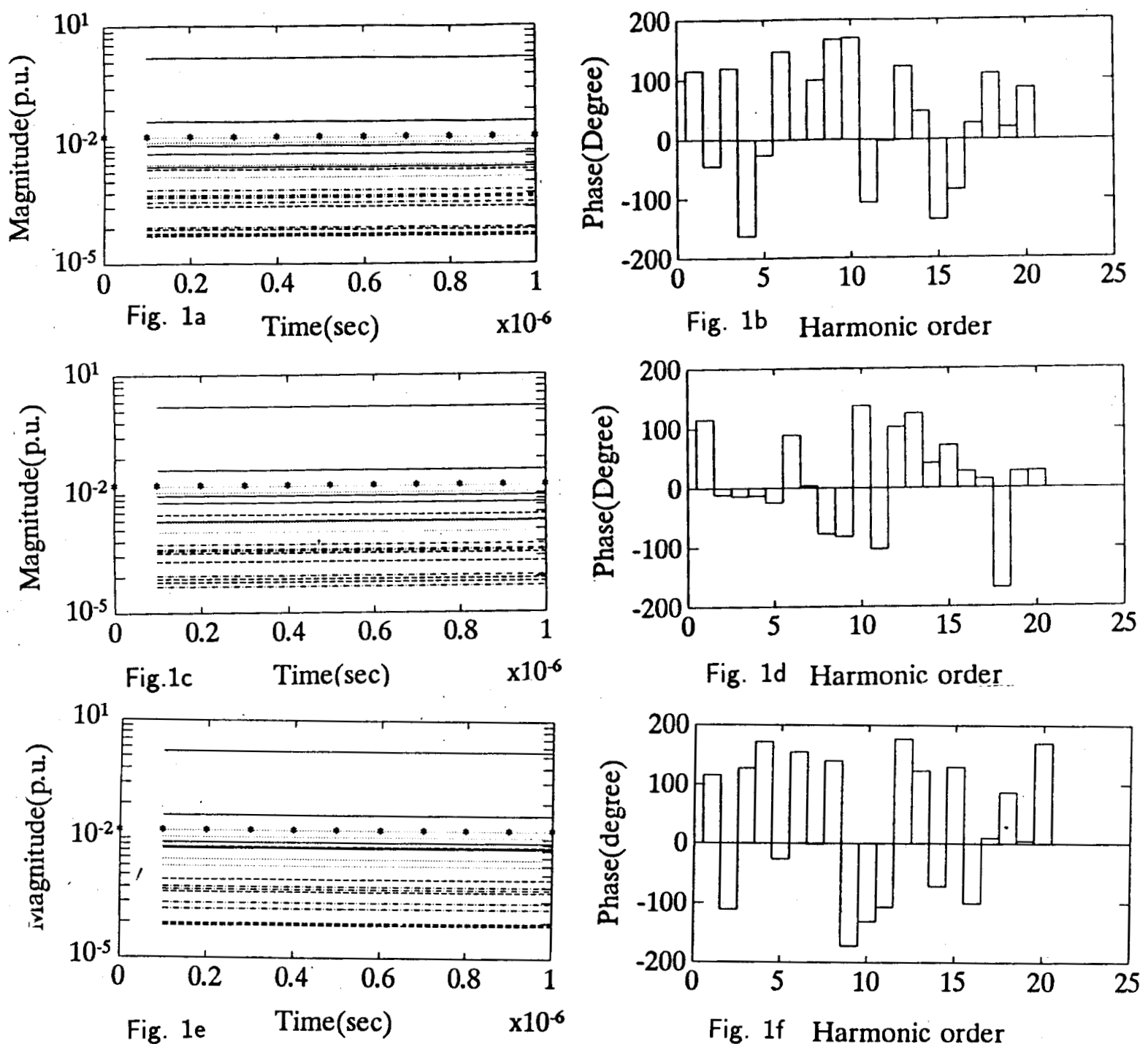

Fig. 1d Harmonic order

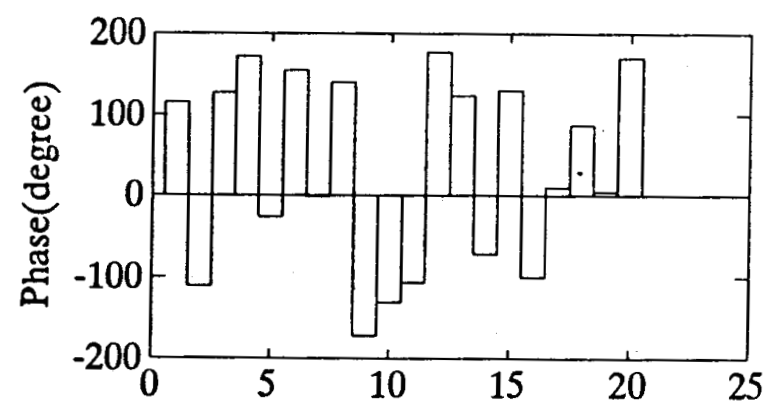

Fig. If Harmonic order
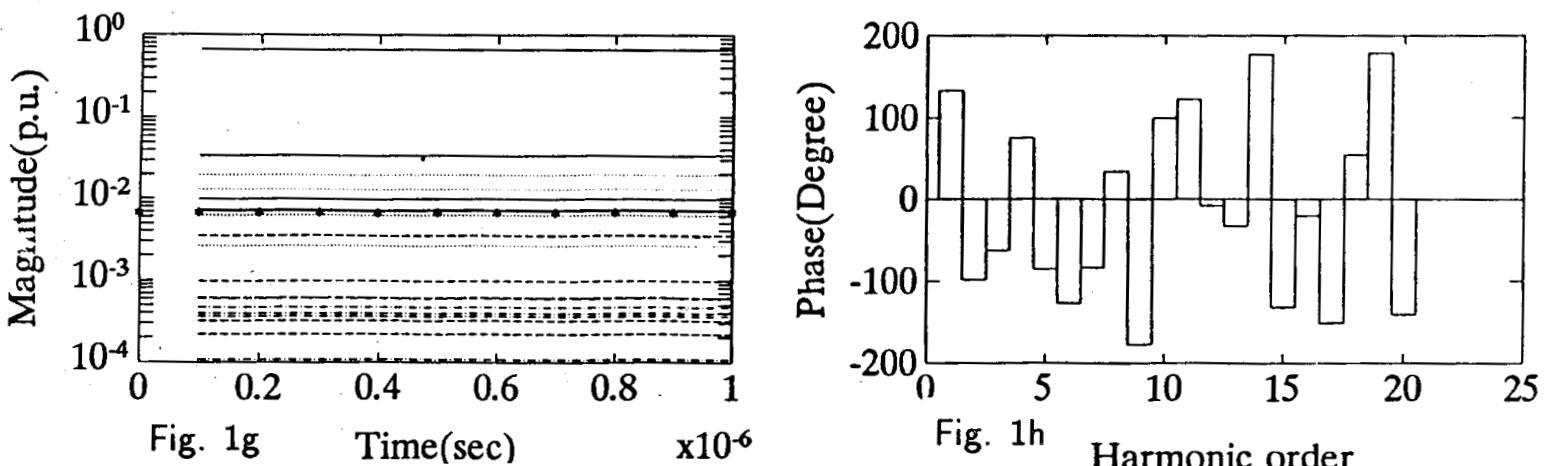

Fig. 1h Harmonic order

Fig 1. ELECTRIC POLLUTION STUDIES OF THE SOURCE CURRENT OF RECTIFIER1 

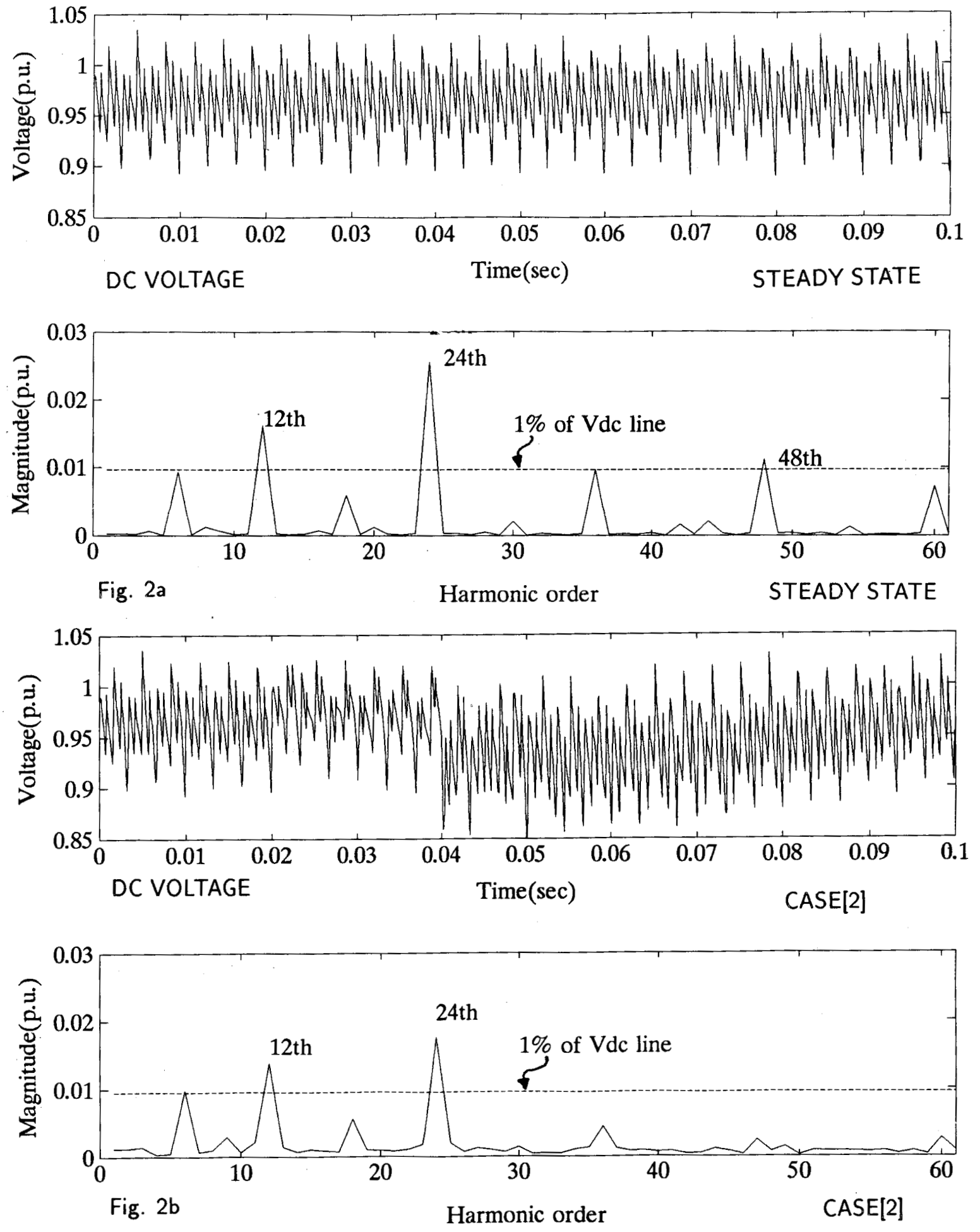

Fig. 2. HARMONIC SPECTRA OF DC VOLTAGE OF RECTIFIER1. 


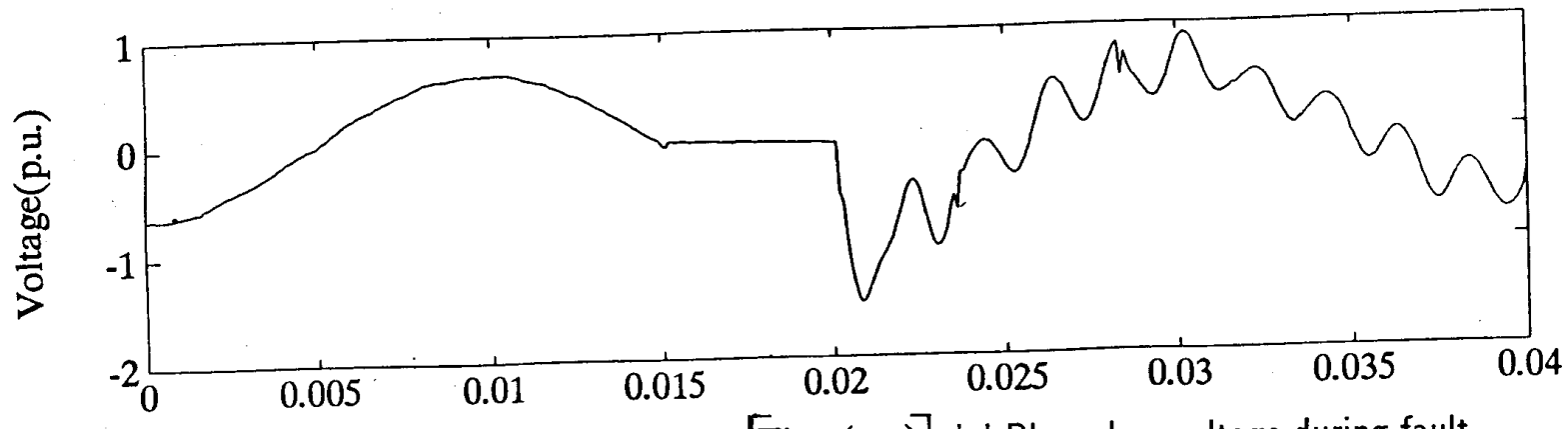

Fig. 3a

$[$ Time(sec)] 'a' Phase bus voltage during fault

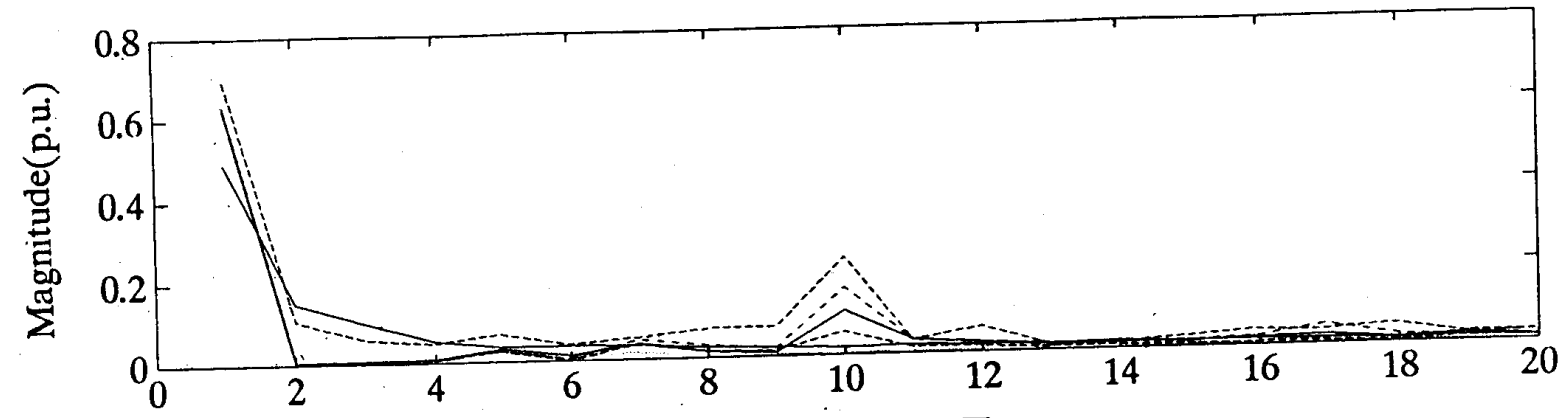

Fig. $3 b$

[Harmonic order] Harmonic spectra of ac bus voltage

Fig. 3 ILLUSTRATION OF VARIATION OF HARMONICS WITH TIME DURING FAULT.
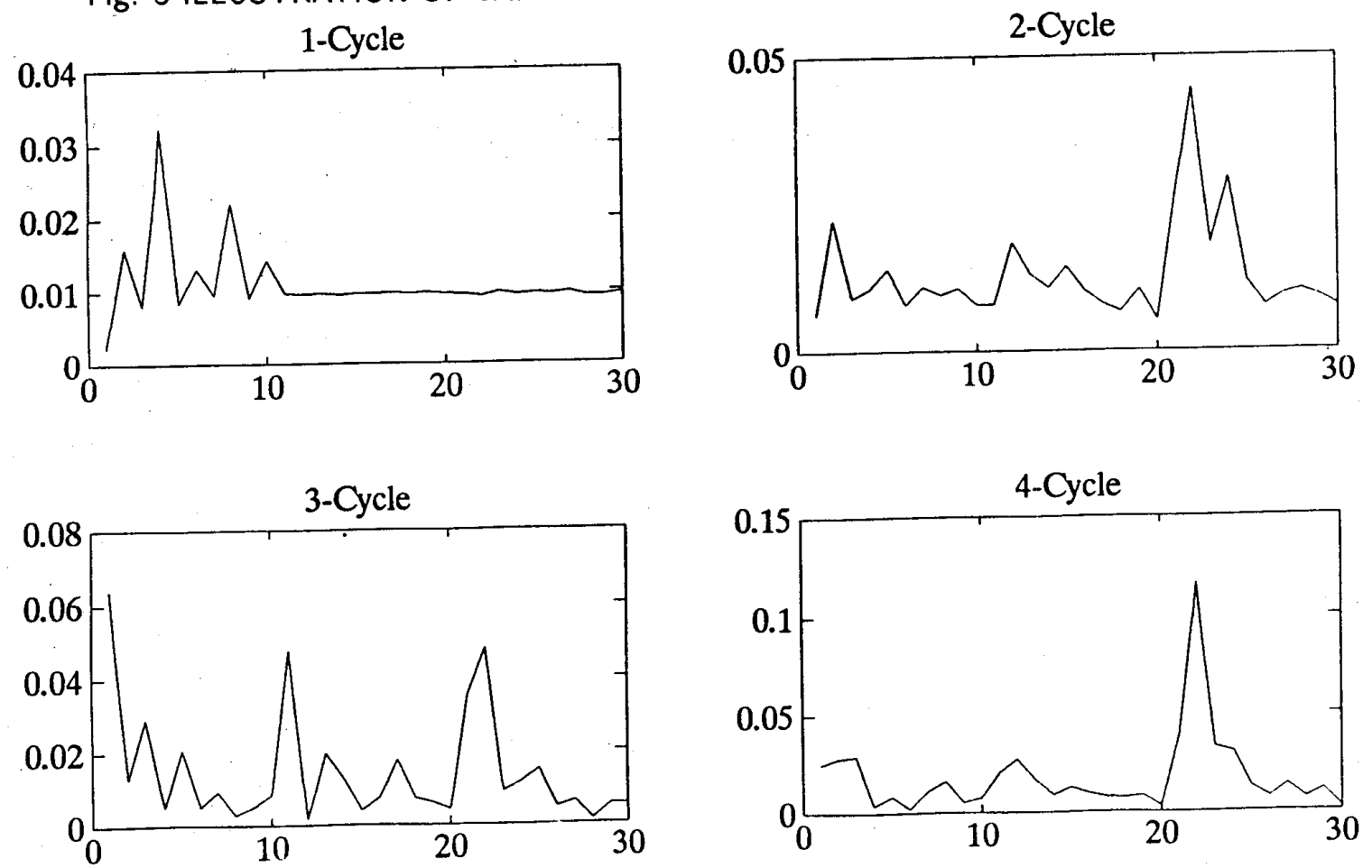

Fig. 4 CHANGE IN THE HARMONIC SPECTRA ON THE DC SIDE DURING FAULT. 